\title{
SOLUTION OF THE DIRICHLET PROBLEM FOR EQUATIONS NOT NECESSARILY STRONGLY ELLIPTIC
}

\author{
BY MARTIN SCHECHTER
}

Communicated by E. Hille, August 7, 1958

Let $\mu=\left(\mu_{1}, \mu_{2}, \cdots, \mu_{n}\right)$ be a sequence of indices and set

$$
\begin{aligned}
|\mu|=\sum \mu_{k}, \quad D^{\mu} & =\partial^{|\mu|} /\left(i \partial x_{1}\right)^{\mu_{1}}\left(i \partial x_{2}\right)^{\mu_{2}} \cdots\left(i \partial x_{n}\right)^{\mu_{n}}, \\
\xi^{\mu} & =\xi_{1}^{\mu_{1} \xi_{2}^{\mu_{2}}} \cdots \xi_{n}^{\mu_{n}}
\end{aligned}
$$

where $\xi=\left(\xi_{1}, \xi_{2}, \cdots, \xi_{n}\right)$ is any $n$-dimensional vector. The linear partial differential operator

$$
A=\sum_{|\mu| \leqq m} a_{\mu}(x) D^{\mu}
$$

with complex coefficients $a_{\mu}$ is elliptic at a point $x$ if

$$
P(x, \xi) \equiv \sum_{|\mu|=m} a_{\mu}(x) \xi^{\mu} \neq 0
$$

for all real $\xi \neq 0$. It is strongly elliptic there if there is a complex constant $\gamma$ such that $\operatorname{Re} \gamma P(x, \xi) \neq 0$ for $\xi \neq 0$. Let $G$ be a bounded domain in $n$-space and let $f$ and $u_{0}$ be smooth complex functions defined in $G$. The Dirichlet problem $\left(A, f, u_{0}\right)$ is to find a complex function $u$ such that $A u=f$ in $G$ and all derivatives of $u-u_{0}$ of order $<m / 2$ vanish on the boundary $\dot{G}$ of G. Gårding [2] and others have shown that if $\dot{G}$ and the coefficients $a_{\mu}$ are sufficiently smooth, a unique solution exists provided $A$ is strongly elliptic and $a_{00} \ldots 0$ is large enough.

In this paper we extend the existence theory to include any elliptic operator for $n>2$ and to operators satisfying a root condition [5] if $n=2$. Such operators will be called properly elliptic. For $m=2$ all properly elliptic operators are strongly elliptic, but this is not the case for higher orders. For example, the operator corresponding to

$$
P(x, \xi)=\xi_{1}^{4}+\xi_{2}^{4}-\xi_{3}^{4}+i\left(\xi_{1}^{2}+\xi_{2}^{2}\right) \xi_{3}^{2}
$$

is not strongly elliptic.

THEOREM. Let $A$ be properly elliptic and denote its formal adjoint by $A^{*}$. Assume that the Dirichlet problem $\left(A^{*}, 0,0\right)$ has only the solution 
$u \equiv 0$. Then for any $f$ and $u_{0}$ sufficiently smooth the Dirichlet problem $\left(A, f, u_{0}\right)$ has a solution.

Sketch of Proof. Without loss of generality, we may assume $u_{0} \equiv 0$ and for convenience we assume $f \in C^{\infty}(\bar{G})$. Set

$$
(v, w)_{s}=\sum_{|\mu| \leqq s} \int_{G} D^{\mu_{v}} \overline{D^{\mu} w} d x \quad\|v\|_{s}^{2}=(v, v)_{s}
$$

and let $V$ be the set of all $v \in C^{\infty}(\bar{G})$ having all derivatives of order $<m / 2$ vanishing on $\dot{G}$. Complete $V$ with respect to the norm \|\|$_{m}$ and call the resulting Hilbert space $H$. From the assumptions on $A$ and $A^{*}$ it follows [5] that

$$
c^{-1}\|v\|_{m} \leqq\left\|A^{*} v\right\|_{0} \leqq c\|v\|_{m} \quad \text { for all } v \in H .
$$

Hence, by the Lax-Milgram lemma [3] there is a $g \in H$ such that

$$
\left(A^{*} g, A^{*} v\right)_{0}=(f, v)_{0} \quad \text { for all } v \in H .
$$

Applying the regularity theory of Nirenberg [4] and Browder [1], we see that $g \in C^{\infty}(\bar{G})$. Hence $A A^{*} g=f$ in $G$. Set $u=A^{*} g \in C^{\infty}(\bar{G})$. Then $A u=f$ in $G$ and

$$
\left(u, A^{*} v\right)_{0}=(A u, v)_{0} \quad \text { for all } v \in H .
$$

This last equality implies $u \in H$. The proof is thus complete.

The foregoing method can also be applied to systems of equations and to general boundary problems which cover $A$ in the sense of [6].

\section{REFERENCES}

1. F. E. Browder, On the regularity properties of solutions of elliptic differential equations, Comm. Pure Appl. Math. vol. 9 (1956) pp. 351-361.

2. Lars Gårding, Dirichlet's problem for linear elliptic partial differential equations, Math. Scand. vol. 1 (1953) pp. 55-72.

3. P. D. Lax and A. N. Milgram, Parabolic equations, Annals of Mathematical Studies, no. 33, 1954, pp. 167-190.

4. Louis Nirenberg, Remarks on strongly elliptic partial differential equations, Comm. Pure Appl. Math. vol. 8 (1955) pp. 648-674.

5. Martin Schechter, On estimating elliptic partial differential operators in the $L_{2}$ norm, Amer. J. Math. vol. 79 (1957) pp. 431-443.

6. - Integral inequalities for partial differential operators and functions satisfying general boundary conditions, Comm. Pure Appl. Math. to appear in 1959.

NEW YoRK UNIVERsity 